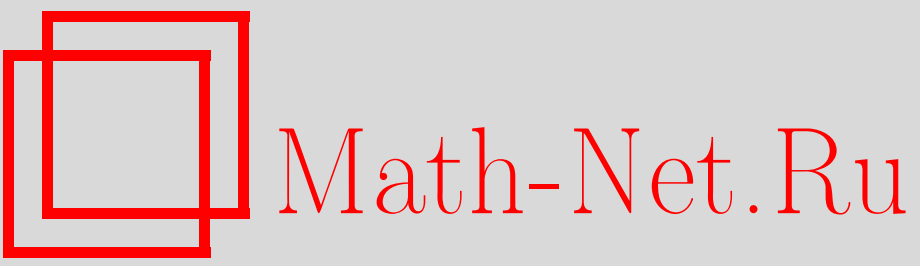

С. Е. Сысоев, Единственность восстановления суммируемой в полосе функции по ее интегралам по окружностям с центрами на фиксированной прямой, $У M H$, 1997, том 52, выпуск 4, 213-214

DOI: https://doi.org/10.4213/rm875

Использование Общероссийского математического портала Math-Net.Ru подразумевает, что вы прочитали и согласны с пользовательским соглашением

http://www.mathnet.ru/rus/agreement

Параметры загрузки :

IP: 52.23 .180 .231

26 апреля 2023 г., 13:21:00 


\title{
ЕДИНСТВЕННОСТЬ ВОССТАНОВЛЕНИЯ СУММИРУЕМОЙ В ПОЛОСЕ ФУНКЦИИ ПО ЕЕ ИНТЕГРАЛАМ ПО ОКРУЖНОСТЯМ С ЦЕНТРАМИ НА ФИКСИРОВАННОЙ ПРЯМОЙ
}

\author{
C. Е. СыCOEB
}

В полосе $\Omega=\{(\xi, \eta): \xi \in \mathbb{R}, 0<\eta<h\}$ рассмотрим семейство дуг окружностей $\gamma(x, y)$ : $\eta=\sqrt{(y+c)^{2}-(\xi-x)^{2}}-c, \xi \in\left(\xi_{1}, \xi_{2}\right)$, с концами $\xi_{1}, \xi_{2}$ на прямой $\eta=0(c=$ const $>0)$. Для непрерывной финитной функции $u \in C_{0}(\Omega)$ положим $(P u)(x, y)=\int_{\gamma(x, y)} u(\xi, \eta) d s=g(x, y)$, где $d s$ - евклидов элемент длины кривой $\gamma$. Требуется по функции $P u$, заданной в $\Omega$, восстановить функцию $u$. В [1] приведено доказательство единственности решения уравнения $P u=g$ в классе непрерывных функций с носителем в верхней полуплоскости. По непрерьвности распространим оператор $P$ на пространство $L_{1}(\Omega)$ суммируемых в полосе $\Omega$ функций. Следуя методу, изложенному в [2], докажем следующую теорему.

Tеорема. Решение уравнения $P u=g$ единственно в $L_{1}(\Omega)$.

Рассмотрим семейство дуг гипербол $\gamma^{*}(\xi, \eta): y=\sqrt{(\eta+c)^{2}+(\xi-x)^{2}}-c, x \in\left(x_{1}, x_{2}\right)$, где $x_{1}, x_{2}$ - точки пересечения гиперболы $(y+c)^{2}-(x-\xi)^{2}=(\eta+c)^{2}$ в плоскости $(x, y)$ с прямой $y=h$. Для $v \in C_{0}(\Omega)$ положим $\left(P^{*} v\right)(\xi, \eta)=\int_{x_{1}}^{x_{2}} v\left(x, \sqrt{(x-\xi)^{2}+(\eta+c)^{2}}-c\right) d x$; тогда для любых $u, v \in C_{0}(\Omega)$ имеет место $\langle P u, v\rangle=\left\langle u, P^{*} v\right\rangle$, где $\langle\cdot, \cdot\rangle$ - скалярное произведение в $L_{2}(\Omega)$. По непрерывности эта формула распространяется на $u \in L_{1}(\Omega), v \in C(\Omega) \cap$ $L_{\infty}(\Omega)$. Рассмотрим отображения $\varphi_{j}: \Omega \rightarrow \Omega_{j}, j=1,2$, задаваемые формулами $\varphi_{j}(\xi, \eta)=$ $\left(x_{j}(\xi, \eta), t_{j}(\xi, \eta)\right)$, где $\Omega_{1}=\mathbb{R} \times(-1,0), \Omega_{2}=\mathbb{R} \times(0,1), x_{j}(\xi, \eta)=\xi+(-1)^{j} \sqrt{(h+c)^{2}-(\eta+c)^{2}}$, $t_{j}(\xi, \eta)=(-1)^{j} \frac{\sqrt{(h+c)^{2}-(\eta+c)^{2}}}{h+c}$. Отображение $\varphi_{j}$ сопоставляет вершине $(\xi, \eta)$ дуги гиперболы $\gamma^{*}(\xi, \eta)$ один из ееконцов $x_{j}$ и тангенс угла наклона $\gamma^{*}$ в этой точке. Пусть $w(x, y, t)$ - решение задачи Коши

$$
L w \equiv t \frac{\partial w}{\partial y}+\frac{\partial w}{\partial x}+\frac{(\eta+c)^{2}}{(y+c)^{3}} \frac{\partial w}{\partial t}=v(x, y), \quad w(x, h, t)=f(x, t) .
$$

Вдоль кривой $\gamma^{*}(\xi, \eta) \quad(L w)\left(x, y(x), y^{\prime}(x)\right)=\frac{d}{d x} w\left(x, y(x), y^{\prime}(x)\right)$, поэтому $\left(P^{*} v\right)(\xi, \eta)=$ $w\left(x_{2}, y\left(x_{2}\right), y^{\prime}\left(x_{2}\right)\right)-w\left(x_{1}, y\left(x_{1}\right), y^{\prime}\left(x_{1}\right)\right)=f\left(x_{2}(\xi, \eta), t_{2}(\xi, \eta)\right)-f\left(x_{1}(\xi, \eta), t_{1}(\xi, \eta)\right)$, где $Q f(\xi, \eta)=f\left(\varphi_{2}(\xi, \eta)\right)-f\left(\varphi_{1}(\xi, \eta)\right)$. Значит, $\langle P u, v\rangle=\langle u, Q f\rangle$, где $v$ связана $\mathrm{c} f$ посредством (1). Найдем сопряженный оператор $Q^{*}$. Пусть $u \in C_{0}(\Omega), \chi_{j}$ - характеристическая функция множества $\Omega_{j}$; тогда

$$
\begin{aligned}
& \langle u, Q f\rangle_{L_{2}(\Omega)}=\sum_{j=1}^{2}(-1)^{j} \int_{\Omega} u(\xi, \eta) f\left(\varphi_{j}(\xi, \eta)\right) d \xi d \eta \\
& \quad=(h+c) \int_{\Omega_{1} \cup \Omega_{2}} \sum_{j=1}^{2}(-1)^{j} \chi_{j}(x, t) u\left(\varphi_{j}^{-1}(x, t)\right) f(x, t) \frac{|t|}{\sqrt{1-t^{2}}} d x d t=\left\langle Q^{*} u, f\right\rangle_{L_{2}\left(\Omega_{1} \cup \Omega_{2}\right)},
\end{aligned}
$$

где

$$
Q^{*} u(x, t)=(h+c) \sum_{j=1}^{2}(-1)^{j} \chi_{j}(x, t) u\left(\varphi_{j}^{-1}(x, t)\right) \frac{|t|}{\sqrt{1-t^{2}}},
$$

причем $\left\|Q^{*} u\right\|_{L_{1}\left(\Omega_{1} \cup \Omega_{2}\right)}=2\|u\|_{L_{1}(\Omega)}$, следовательно, $Q^{*} / 2$ - изометрический оператор, действующий из $L_{1}(\Omega)$ в $L_{1}\left(\Omega_{1} \cup \Omega_{2}\right)$. Следовательно, верна

Лемма. Для любых $u \in L_{1}(\Omega), f \in C\left(\overline{\Omega_{1} \cup \Omega_{2}}\right)$ справедливо равенство $\langle P u, v\rangle=$ $\left\langle Q^{*} u, f\right\rangle$, где функция $v \in C(\bar{\Omega})$ связана с $f$ соотношениями (1), а оператор $Q^{*}$ определен формулой (2). При этом $\left\|Q^{*} u\right\|_{1}=2\|u\|_{1}$, где $\|\cdot\|_{1}-$ норма в $L_{1}(\Omega)$. 
Полагая в (1) $t=0$, получим $v(x, y)=\frac{\partial w}{\partial x}(x, y, 0)+\frac{(\eta+c)^{2}}{(y+c)^{3}} \frac{\partial w}{\partial t}(x, y, 0)$, следовательно, $\frac{\partial w}{\partial y}(x, y, t)=-D\left(\frac{\partial w}{\partial x}+\frac{(\eta+c)^{2}}{(y+c)^{3}} \frac{\partial w}{\partial t}\right)(x, y, t)$, где $D\left(\frac{\partial w}{\partial x}+\frac{(\eta+c)^{2}}{(y+c)^{3}} \frac{\partial w}{\partial t}\right)(x, y, t):=\frac{1}{t}\left(\left(\frac{\partial w}{\partial x}+\frac{(\eta+c)^{2}}{(y+c)^{3}} \frac{\partial w}{\partial t}\right)(x, y, t)-\left(\frac{\partial w}{\partial x}+\frac{(\eta+c)^{2}}{(y+c)^{3}} \frac{\partial w}{\partial t}\right)(x, y, 0)\right)$. Интегрируя $\frac{\partial w}{\partial y}$ по $y$ от $y$ до $h$, получим $w(x, h, t)-w(x, y, t)=f(x, t)-w(x, y, t)=-\int_{y}^{h} D\left(\frac{\partial w}{\partial x}+\frac{(\eta+c)^{2}}{(y+c)^{3}} \frac{\partial w}{\partial t}\right)(x, y, t) d y=-V w$, т.е. $w=V w+f$. Функцию $f(x, t)$ можно считать заданной в полосе $\mathbb{R} \times(-1,1)$. Обозначим $E_{s}=\left\{(x, t) \in \mathbb{C}^{2}:|\operatorname{Im} x|<\varepsilon(1+s),|t|<1+\varepsilon(1+s)\right\}$, где $s \in[0,1]$ и $\varepsilon>0$ - произвольное фиксированное. В качестве банахова пространства $X_{s}$ возьмем пространство функций $f(x, t)$, аналитических в области $E_{s}$ и ограниченных в ее замькании. Положим $\|f\|_{s}=\sup _{E_{s}}|f(x, t)|$. При $s^{\prime}<s \quad X_{s} \subseteq X_{s^{\prime}},\|f\|_{s^{\prime}} \leqslant\|f\|_{s}$ для всех $f \in X_{s}$. Таким образом, линейное пространство $\Xi=\bigcup X_{s}, s \in[0,1],-$ шкала банаховых пространств. По банахову пространству $C[0, h]$ и шкале $\Xi$ естественным образом строится шкала

$$
C([0, h] ; \Xi)=\bigcup_{s \in[0,1]} C\left([0, h] ; X_{s}\right), \quad\|w\|_{C\left([0, h] ; X_{s}\right)}=\sup _{y \in[0, h]}\|w(\cdot, y, \cdot)\|_{s}
$$

где $C\left([0, h] ; X_{s}\right)$ - банахово пространство функций $w:[0, h] \rightarrow X_{s}$, непрерьвных на $[0, h]$. Для $s^{\prime}<s$ справедлива оценка

$$
\left\|D\left(\frac{\partial w}{\partial x}+\frac{(\eta+c)^{2}}{(y+c)^{3}} \frac{\partial w}{\partial t}\right)\right\|_{s^{\prime}} \leqslant \frac{2}{\varepsilon\left(s-s^{\prime}\right)}\left(1+\frac{(h+c)^{2}}{c^{3}}\right)\|w\|_{s},
$$

следовательно, $\|V w\|_{s^{\prime}} \leqslant \frac{c_{1}}{s-s^{\prime}} \int_{y}^{h}\|w(\cdot, y, \cdot)\|_{s} d y$, где $c_{1}=\frac{2}{\varepsilon}\left(1+\frac{(h+c)^{2}}{c^{3}}\right)$. Рассмотрим линейньй ограниченньй оператор $A: C[0, h] \rightarrow C[0, h]$, действующий по формуле $(A \varphi)(y)=$ $c_{1} \int_{y}^{h} \varphi(\eta) d \eta, \varphi \in C[0, h]$. У целой функции $f_{A}(\lambda)=\sum_{n=0}^{\infty}\left\|A^{n}\right\| \lambda^{n}, \lambda \in \mathbb{C}$, порядок $\rho=1$, тип $\sigma=c_{1} h$, поэтому если $е c_{1} h<1$, то при $f \in X_{1}$ существует решение $w \in C\left([0, h] ; X_{0}\right)$ уравнения $w=V w+f\left[2\right.$, теорема 1.1 для шкалы $C\left([0, h] ; X_{s}\right)$, с. 79]. Продельвая вькладки, приведшие к этому уравнению, в обратном порядке и полагая $v(x, y)=\left(\frac{\partial w}{\partial x}+\frac{(\eta+c)^{2}}{(y+c)^{3}} \frac{\partial w}{\partial t}\right)(x, y, 0)$, приходим к (1).

ДоКАЗАТЕЛЬСТво тЕОРЕМЫ. Выбирая $\varepsilon>0$ и уменьшая, если нужно, $h$, добьемся выполнения неравенства $e c_{1} h<1$. Тогда для $f \in X_{1}$ существует $v \in C(\bar{\Omega})$ такая, что $\langle P u, v\rangle=$ $\left\langle Q^{*} u, f\right\rangle$. При $P u=0$ получаем $\left\langle Q^{*} u, f\right\rangle=0$ для всех $f \in X_{1}$, следовательно, $Q^{*} u=0$ в силу плотности аналитических функций в пространстве $C\left(\overline{\Omega_{1} \cup \Omega_{2}}\right)$. По лемме $\left\|Q^{*} u\right\|_{1}=2\|u\|_{1}$, следовательно, $u=0$ почти всюду в полосе $\{(x, y): x \in \mathbb{R}, 0 \leqslant y \leqslant h\}$. Повторяя эти рассуждения для $[h, 2 h],[2 h, 3 h]$ и т. д., получаем теорему единственности для любого конечного $h$.

Автор глубоко благодарен своему научному руководителю д.ф.-м.н., профессору В. П. Паламодову за внимание к работе и полезные обсуждения.

\section{СПИСОК ЛИТЕРАТУРЫ}

[1] Курант Р. Уравнения с частными производными. М.: Мир, 1964. [2] Бухгейм А. Л. Введение в теорию обратных задач. Новосибирск: Наука, 1988. 